Benefits of combination pharmacotherapy for HFrEF

In patients with heart failure with reduced ejection fraction (HFrEF), $\beta$-blockers, mineralocorticoidreceptor antagonists (MRAs) and sodium-glucose cotransporter 2 (SGLT2) inhibitors have shown benefit in placebo-controlled trials, and angiotensin receptor-neprilysin inhibitors (ARNIs) provide incremental benefit compared with angiotensin-converting enzyme (ACE) inhibitors. However, because these agents were generally tested in parallel and not incrementally, the additive benefits of combining these therapies are untested. Moreover, ARNIs, SGLT2 inhibitors and MRAs remain suboptimally prescribed in clinical practice. In a new cross-trial analysis, researchers predict that a comprehensive strategy combining several drugs reduces hospitalization and mortality compared with conventional therapy.
There are incremental benefits of treating patients with HFrEF with all four agents
The analysis included patient-level data from three randomized, controlled trials (EMPHASIS-HF, PARADIGM-HF and DAPA-HF) with a combined total of 15,880 patients with HFrEF (78\% men). Compared with conventional therapy (a $\beta$-blocker and an ACE inhibitor or angiotensin-receptor blocker), a comprehensive strategy (combining an ARNI, $\beta$-blocker, MRA and SGLT2 inhibitor) was predicted to reduce the primary end point of cardiovascular death or hospitalization for heart failure (HR 0.38, 95\% CI 0.30-0.47) as well as each of these end point individually and all-cause mortality. With the comprehensive strategy, a patient aged 80 years was estimated to gain 2.7 additional years free from a primary end point event and would survive for an additional

1.4 years. These outcomes increased to 8.3 and 6.3 additional years, respectively, for a patient aged 55 years.

"There are incremental benefits of treating patients with HFrEF with all four agents impacting five pathways (angiotensin II, aldosterone, noradrenaline, neprilysin and SGLT2), and this approach should be considered the standard of care,"

\title{
ARRHYTHMIAS
}

\section{A fully biological defibrillation system for restoring cardiac rhythm}

Once cardiac electrical activity is disturbed, such as with sustained arrhythmias, the heart is not capable of self-restoring a normal rhythm. However, according to a new study combining theoretical and experimental assays, a biological detector-effector system involving the expression of a customized ion channel in cardiomyocytes can enable the heart to automatically detect and terminate acute arrhythmias. Studies on these biological self-restoring systems can contribute to the development of arrhythmia management strategies that avoid the need for devices, wires or electric shocks for defibrillation.

The biological self-restoring system developed by the group of Daniël Pijnappels, named the biologically integrated cardiac defibrillator (BiolCD), involves the introduction of a new ion channel with specific properties into the sarcolemma of cardiomyocytes. The gating of the ion channel is customized to differentiate between normal rhythm and arrhythmia (based on the detection of high-frequency signals irrespective of the nature and underlying cause of the arrhythmia) and to generate an ionic current for the termination of the detected arrhythmia. First, the investigators validated the BioICD concept in silico using simulations of human cardiomyocyte monolayers and of atria and ventricles. The virtual models that had the BiolCD integrated were capable of autodetecting the arrhythmia and restoring sinus rhythm within seconds of arrhythmia initiation without the need of a non-biological factor. Next, the researchers validated the BiolCD concept in living cells in vitro. The experimental assays involved the dynamic patch-clamp technique (which allows the introduction of virtual voltagegated ion channels, such as the BioICD, into excitable cells) and human atrial cardiomyocytes optogenetically

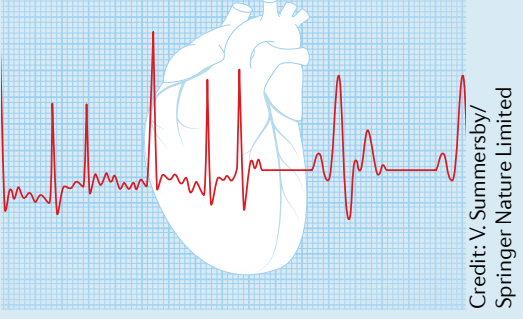

modified to express a light-gated ion channel (which enables optical control of excitation rhythm with a light source). The data showed that the same ion channel gating properties used for the computer simulations enabled the detection of arrhythmias and the restoration of cardiac excitation rhythm in the living cells.

Taken together, these findings provide proof of concept of a fully biological detector-effector system for the regulation of cardiac arrhythmias.

Irene Fernández-Ruiz

ORIGINAL ARTICLE Majumder, R. et al.

Self-restoration of cardiac excitation rhythm by anti-arrhythmic ion channel gating. eLife $\mathbf{9}$, e55921 (2020)

RELATED ARTICLE Niederer, S. A. et al. Computational models in cardiology. Nat. Rev. Cardiol. 16, 100-111 (2020) 